\title{
Delay-Partitioning Approach to Stability of Linear Discrete-Time Systems with Interval-Like Time-Varying Delay
}

\author{
Priyanka Kokil, V. Krishna Rao Kandanvli, and Haranath Kar \\ Department of Electronics and Communication Engineering, Motilal Nehru National Institute of Technology Allahabad, \\ Allahabad 211004, India \\ Correspondence should be addressed to Priyanka Kokil; kokilnit@gmail.com
}

Received 19 March 2013; Accepted 26 May 2013

Academic Editor: J. A. Tenreiro Machado

Copyright (C) 2013 Priyanka Kokil et al. This is an open access article distributed under the Creative Commons Attribution License, which permits unrestricted use, distribution, and reproduction in any medium, provided the original work is properly cited.

\begin{abstract}
This paper is concerned with the problem of global asymptotic stability of linear discrete-time systems with interval-like timevarying delay in the state. By utilizing the concept of delay partitioning, a new linear-matrix-inequality-(LMI-) based criterion for the global asymptotic stability of such systems is proposed. The proposed criterion does not involve any free weighting matrices but depends on both the size of delay and partition size. The developed approach is extended to address the problem of global asymptotic stability of state-delayed discrete-time systems with norm-bounded uncertainties. The proposed results are compared with several existing results.
\end{abstract}

\section{Introduction}

A source of instability for discrete-time systems is time delay which inevitably exists in various engineering systems. In many applications, time delays are unavoidable and must be taken into account in a realistic system design, for instance, chemical processes, echo cancellation, local loop equalization, multipath propagation in mobile communication, array signal processing, congestion analysis and control in high speed networks, neural networks, and long transmission line in pneumatic systems [1-6]. The stability analysis of timedelay systems has received considerable attention during the last two decades [3-7]. According to dependence of delay, the existing stability criteria are generally classified into two categories: delay-dependent criteria and delayindependent criteria. It is well known that delay-independent stability criteria are usually more conservative than the delaydependent ones, especially if the size of time delay is small [610]. Therefore, much attention has been paid in recent years to the study of delay-dependent stability criteria.

A number of publications relating to the delay-dependent stability of continuous time-delay systems have appeared (see, e.g., [10-17] and the references cited therein). In contrast, less attention has been paid to studying the problem of stability of discrete time-delay systems. Several delaydependent criteria for the stability of discrete-time systems have appeared [3, 6, 7, 18, 19]. Reference [7] (see [6] also) presents a novel delay-dependent linear-matrix-inequality(LMI-) based condition for the global asymptotic stability of linear discrete-time systems with interval-like time-varying delay. The criteria proposed in $[6,7]$ are less conservative with smaller numerical complexity than [19-23]. The delay partitioning approach has been efficiently applied in [24-30] to the stability analysis of systems with time-varying delays. In the context of stability analysis of linear discrete systems with time-varying delay, the delay partitioning concept is first utilized in [30]. The approaches in $[29,30]$ divide the lower bound of the time-varying delay into a number of partitions. The criteria in $[29,30]$ are not only delay dependent but also dependent on the partitioning size. Though the approaches in $[29,30]$ provide less conservative stability results than $[19$, 20], these approaches would lead to heavier computational burden and more complicated synthesis procedure.

This paper studies the problem of stability analysis of linear discrete-time system with interval-like time-varying delay in the state. In this paper, inspired by the work of $[6,7,30]$, an alternative to the approach presented in [29] for the stability analysis of linear discrete-time systems with 
interval-like time-varying delay in the state is brought out. The proposed method exploits the delay partitioning idea and does not introduce any free weighting matrices. The paper is organized as follows. Section 2 presents a description of the system under consideration. A novel LMI-based criterion for the global asymptotic stability of discrete-time statedelayed systems is proposed in Section 3. The proposed criterion depends on the size of delay as well as partition size. In Section 4, the approach is extended to derive global asymptotic stable conditions for delayed discrete-time systems with norm-bounded uncertainties. Finally, in Section 5, numerical examples are given to illustrate the effectiveness of the presented results.

\section{System Description}

The following notations are used throughout the paper:

$$
\begin{aligned}
& \mathbf{R}^{p \times q} \text { : Set of } p \times q \text { real matrices, } \\
& \mathbf{R}^{p}: \text { Set of } p \times 1 \text { real vectors, }
\end{aligned}
$$

I: Identity matrix of appropriate dimension; the order is specified in subscript as the need arises,

0: Null matrix or null vector of appropriate dimension; the orders are specified in subscripts as the need arises,

$\mathbf{B}^{T}$ : Transpose of the matrix (or vector) $\mathbf{B}$,

B > 0: $\mathbf{B}$ is positive-definite symmetric matrix,

$\mathbf{B}<\mathbf{0}$ : $\mathbf{B}$ is negative-definite symmetric matrix.

The system under consideration is given by

$$
\begin{gathered}
\mathbf{x}(k+1)=\mathbf{A} \mathbf{x}(k)+\mathbf{A}_{1} \mathbf{x}(k-d(k)), \\
\mathbf{x}(k)=\phi(k), \quad k=-h_{2},-h_{2}+1, \ldots, 0,
\end{gathered}
$$

where $\mathbf{x}(k) \in \mathbf{R}^{n}$ is the system state vector, $\mathbf{A}$ and $\mathbf{A}_{1}$ are constant matrices with appropriate dimensions, $d(k)$ is a positive integer representing interval-like time-varying delay satisfying

$$
1 \leq h_{1} \leq d(k) \leq h_{2}
$$

where $h_{1}$ and $h_{2}$ are known positive integers representing the lower and upper delay bounds, respectively, and $\phi(k)$ is an initial value at time $k$. Let the lower bound of the delay $h_{1}$ be divided into $m$ number of partitions such that

$$
h_{1}=\tau m
$$

where $\tau$ is an integer representing partition size.

\section{Proposed Criterion}

In this section, inspired by the work of $[6,7,30]$, an LMIbased criterion for the global asymptotic stability of system (1)-(4) is established.

The main result may be stated as follows.
Theorem 1. For given positive integers $\tau, m$, and $h_{2}$, the system in (1)-(4) is asymptotically stable if there exist real matrices $\mathbf{P}=$ $\mathbf{P}^{T}>\mathbf{0}, \mathbf{Q}_{i}=\mathbf{Q}_{i}^{T}>\mathbf{0}(i=1,2,3)$, and $\mathbf{Z}_{i}=\mathbf{Z}_{i}^{T}>\mathbf{0}(i=1,2)$ such that

$$
\begin{aligned}
\boldsymbol{\Psi}_{1}= & \boldsymbol{\Psi}-\left[\begin{array}{llll}
\mathbf{0}_{n \times m n} & \mathbf{I}_{n} & -\mathbf{I}_{n} & \mathbf{0}_{n}
\end{array}\right]^{T} \mathbf{Z}_{2} \\
& \times\left[\begin{array}{llll}
\mathbf{0}_{n \times m n} & \mathbf{I}_{n} & -\mathbf{I}_{n} & \mathbf{0}_{n}
\end{array}\right]<\mathbf{0}, \\
\boldsymbol{\Psi}_{2}= & \boldsymbol{\Psi}-\left[\begin{array}{lll}
\mathbf{0}_{n \times(m+1) n} & \mathbf{I}_{n} & -\mathbf{I}_{n}
\end{array}\right]^{T} \mathbf{Z}_{2} \\
& \times\left[\begin{array}{lll}
\mathbf{0}_{n \times(m+1) n} & \mathbf{I}_{n} & -\mathbf{I}_{n}
\end{array}\right]<\mathbf{0},
\end{aligned}
$$

where

$$
\begin{aligned}
& \boldsymbol{\Psi}=\boldsymbol{\Lambda}_{1}^{T} \boldsymbol{\Phi}_{1} \boldsymbol{\Lambda}_{1}+\boldsymbol{\Lambda}_{2}^{T} \boldsymbol{\Phi}_{2} \boldsymbol{\Lambda}_{2}+\boldsymbol{\Lambda}_{3}^{T} \boldsymbol{\Phi}_{3} \boldsymbol{\Lambda}_{3}-\boldsymbol{\Lambda}_{4}^{T} \boldsymbol{\Phi}_{4} \boldsymbol{\Lambda}_{4}+\boldsymbol{\Lambda}_{1}^{T} \boldsymbol{\Phi}_{5} \boldsymbol{\Lambda}_{3} \\
& +\boldsymbol{\Lambda}_{3}^{T} \boldsymbol{\Phi}_{5}^{T} \boldsymbol{\Lambda}_{1}-\boldsymbol{\Lambda}_{5}^{T} \mathbf{Z}_{1} \boldsymbol{\Lambda}_{5}+\boldsymbol{\Lambda}_{1}^{T} \mathbf{Z}_{1} \boldsymbol{\Lambda}_{5}+\boldsymbol{\Lambda}_{5}^{T} \mathbf{Z}_{1} \boldsymbol{\Lambda}_{1}-\boldsymbol{\Lambda}_{6}^{T} \mathbf{Z}_{2} \boldsymbol{\Lambda}_{6} \\
& +\boldsymbol{\Lambda}_{3}^{T} \mathbf{Z}_{2} \boldsymbol{\Lambda}_{4}+\boldsymbol{\Lambda}_{4}^{T} \mathbf{Z}_{2} \boldsymbol{\Lambda}_{3}+\Lambda_{6}^{T} \mathbf{Z}_{2} \boldsymbol{\Lambda}_{3}+\boldsymbol{\Lambda}_{3}^{T} \mathbf{Z}_{2} \boldsymbol{\Lambda}_{6}
\end{aligned}
$$

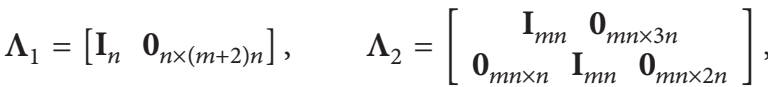

$$
\begin{aligned}
& \boldsymbol{\Lambda}_{3}=\left[\begin{array}{lll}
\mathbf{0}_{n \times(m+1) n} & \mathbf{I}_{n} & \mathbf{0}_{n}
\end{array}\right], \quad \boldsymbol{\Lambda}_{4}=\left[\begin{array}{ll}
\mathbf{0}_{n \times(m+2) n} & \mathbf{I}_{n}
\end{array}\right], \\
& \boldsymbol{\Lambda}_{5}=\left[\begin{array}{lll}
\mathbf{0}_{n} & \mathbf{I}_{n} & \mathbf{0}_{n \times(m+1) n}
\end{array}\right], \quad \boldsymbol{\Lambda}_{6}=\left[\begin{array}{lll}
\mathbf{0}_{n \times m n} & \mathbf{I}_{n} & \mathbf{0}_{n \times 2 n}
\end{array}\right], \\
& \boldsymbol{\Phi}_{1}=\mathbf{A}^{T} \mathbf{P A}-\mathbf{P}+\mathbf{Q}_{2}+\left(h_{2}-\tau m+1\right) \mathbf{Q}_{3}-\mathbf{Z}_{1} \\
& +\left(\mathbf{A}-\mathbf{I}_{n}\right)^{T}\left(\tau^{2} \mathbf{Z}_{1}+\left(h_{2}-\tau m\right)^{2} \mathbf{Z}_{2}\right)\left(\mathbf{A}-\mathbf{I}_{n}\right), \\
& \boldsymbol{\Phi}_{2}=\left[\begin{array}{cc}
\mathbf{Q}_{1} & \mathbf{0} \\
\mathbf{0} & -\mathbf{Q}_{1}
\end{array}\right] \\
& \boldsymbol{\Phi}_{3}=\mathbf{A}_{1}^{T}\left(\mathbf{P}+\tau^{2} \mathbf{Z}_{1}+\left(h_{2}-\tau m\right)^{2} \mathbf{Z}_{2}\right) \mathbf{A}_{1}-\mathbf{Q}_{3}-2 \mathbf{Z}_{2}, \\
& \Phi_{4}=\mathbf{Q}_{2}+\mathbf{Z}_{2} \\
& \Phi_{5}=\mathbf{A}^{T} \mathbf{P} \mathbf{A}_{1}+\left(\mathbf{A}-\mathbf{I}_{n}\right)^{T}\left(\tau^{2} \mathbf{Z}_{1}+\left(h_{2}-\tau m\right)^{2} \mathbf{Z}_{2}\right) \mathbf{A}_{1} .
\end{aligned}
$$

Proof. Consider a Lyapunov function $[29,30]$

$$
\begin{aligned}
& V(k)=\mathbf{x}^{T}(k) \mathbf{P} \mathbf{x}(k)+\sum_{i=k-\tau}^{k-1} \mathbf{Y}^{T}(i) \mathbf{Q}_{1} \mathbf{\Upsilon}(i) \\
& +\sum_{i=k-h_{2}}^{k-1} \mathbf{x}^{T}(i) \mathbf{Q}_{2} \mathbf{x}(i)+\sum_{j=-h_{2}}^{-\tau m} \sum_{i=k+j}^{k-1} \mathbf{x}^{T}(i) \mathbf{Q}_{3} \mathbf{x}(i) \\
& +\sum_{j=-\tau}^{-1} \sum_{i=k+j}^{k-1} \tau \Delta \mathbf{x}^{T}(i) \mathbf{Z}_{1} \Delta \mathbf{x}(i) \\
& +\sum_{j=-h_{2}}^{-\tau m-1} \sum_{i=k+j}^{k-1}\left(h_{2}-\tau m\right) \Delta \mathbf{x}^{T}(i) \mathbf{Z}_{2} \Delta \mathbf{x}(i),
\end{aligned}
$$


where

$$
\begin{gathered}
\mathbf{Y}(i)=\left[\begin{array}{llll}
\mathbf{x}^{T}(i) & \mathbf{x}^{T}(i-\tau) & \cdots & \mathbf{x}^{T}(i-(m-1) \tau)
\end{array}\right]^{T}, \\
\Delta \mathbf{x}(i)=\mathbf{x}(i+1)-\mathbf{x}(i) .
\end{gathered}
$$

Taking the forward difference of (15) along the solution of the system (1)-(2), we have

$\Delta V(k)$

$$
\begin{aligned}
= & V(k+1)-V(k) \\
= & {\left[\mathbf{A x}(k)+\mathbf{A}_{1} \mathbf{x}(k-d(k))\right]^{T} \mathbf{P}\left[\mathbf{A x}(k)+\mathbf{A}_{1} \mathbf{x}(k-d(k))\right] } \\
& -\mathbf{x}^{T}(k) \mathbf{P} \mathbf{x}(k)+\mathbf{Y}^{T}(k) \mathbf{Q}_{1} \mathbf{Y}(k)-\mathbf{Y}^{T}(k-\tau) \mathbf{Q}_{1} \mathbf{\Upsilon}(k-\tau) \\
& +\mathbf{x}^{T}(k) \mathbf{Q}_{2} \mathbf{x}(k)-\mathbf{x}^{T}\left(k-h_{2}\right) \mathbf{Q}_{2} \mathbf{x}\left(k-h_{2}\right) \\
& +\left(h_{2}-\tau m+1\right) \mathbf{x}^{T}(k) \mathbf{Q}_{3} \mathbf{x}(k)+\Delta \mathbf{x}^{T}(k) \tau^{2} \mathbf{Z}_{1} \Delta \mathbf{x}(k) \\
& +\Delta \mathbf{x}^{T}(k)\left(h_{2}-\tau m\right)^{2} \mathbf{Z}_{2} \Delta \mathbf{x}(k)-\sum_{i=k-h_{2}}^{k-\tau m} \mathbf{x}^{T}(i) \mathbf{Q}_{3} \mathbf{x}(i) \\
& -\sum_{i=k-\tau}^{k-1} \tau \Delta \mathbf{x}^{T}(i) \mathbf{Z}_{1} \Delta \mathbf{x}(i) \\
& -\sum_{i=k-h_{2}}^{k-\tau m-1}\left(h_{2}-\tau m\right) \Delta \mathbf{x}^{T}(i) \mathbf{Z}_{2} \Delta \mathbf{x}(i) .
\end{aligned}
$$

Using Lemma 1 in [22], we obtain [6, 7]

$$
\begin{aligned}
& -\sum_{i=k-\tau}^{k-1} \tau \Delta \mathbf{x}^{T}(i) \mathbf{Z}_{1} \Delta \mathbf{x}(i) \\
& \quad \leq-(\mathbf{x}(k)-\mathbf{x}(k-\tau))^{T} \mathbf{Z}_{1}(\mathbf{x}(k)-\mathbf{x}(k-\tau)) .
\end{aligned}
$$

Note that

$$
-\sum_{i=k-h_{2}}^{k-\tau m} \mathbf{x}^{T}(i) \mathbf{Q}_{3} \mathbf{x}(i) \leq-\mathbf{x}^{T}(k-d(k)) \mathbf{Q}_{3} \mathbf{x}(k-d(k)) .
$$

Define

$$
\zeta(k)=\left[\begin{array}{llll}
\mathbf{Y}^{T}(k) & \mathbf{x}^{T}(k-\tau m) & \mathbf{x}^{T}(k-d(k)) & \mathbf{x}^{T}\left(k-h_{2}\right)
\end{array}\right]^{T} .
$$

It follows from (1) and (17) that

$$
\Delta \mathbf{x}(k)=\left(\mathbf{A}-\mathbf{I}_{n}\right) \mathbf{x}(k)+\mathbf{A}_{1} \mathbf{x}(k-d(k)) .
$$

Now, we have the following relation $[6,7]$ :

$$
\begin{aligned}
- & \sum_{i=k-h_{2}}^{k-\tau m-1}\left(h_{2}-\tau m\right) \Delta \mathbf{x}^{T}(i) \mathbf{Z}_{2} \Delta \mathbf{x}(i) \\
= & -\sum_{i=k-h_{2}}^{k-d(k)-1}\left(h_{2}-\tau m\right) \Delta \mathbf{x}^{T}(i) \mathbf{Z}_{2} \Delta \mathbf{x}(i) \\
& -\sum_{i=k-d(k)}^{k-\tau m-1}\left(h_{2}-\tau m\right) \Delta \mathbf{x}^{T}(i) \mathbf{Z}_{2} \Delta \mathbf{x}(i) \\
= & -\sum_{i=k-h_{2}}^{k-d(k)-1}\left(h_{2}-d(k)\right) \Delta \mathbf{x}^{T}(i) \mathbf{Z}_{2} \Delta \mathbf{x}(i) \\
& -\sum_{i=k-h_{2}}^{k-d(k)-1}(d(k)-\tau m) \Delta \mathbf{x}^{T}(i) \mathbf{Z}_{2} \Delta \mathbf{x}(i) \\
& -\sum_{i=k-d(k)}^{k-\tau m-1}\left(h_{2}-d(k)\right) \Delta \mathbf{x}^{T}(i) \mathbf{Z}_{2} \Delta \mathbf{x}(i) \\
& -\sum_{i=k-d(k)}^{k-\tau m-1}(d(k)-\tau m) \Delta \mathbf{x}^{T}(i) \mathbf{Z}_{2} \Delta \mathbf{x}(i) .
\end{aligned}
$$

Letting $\beta=(d(k)-\tau m) /\left(h_{2}-\tau m\right)$, one obtains [6]

$$
-\sum_{i=k-h_{2}}^{k-d(k)-1}(d(k)-\tau m) \Delta \mathbf{x}^{T}(i) \mathbf{Z}_{2} \Delta \mathbf{x}(i)
$$

$$
\begin{aligned}
& \quad=-\beta \sum_{i=k-h_{2}}^{k-d(k)-1}\left(h_{2}-\tau m\right) \Delta \mathbf{x}^{T}(i) \mathbf{Z}_{2} \Delta \mathbf{x}(i) \\
& \leq-\beta \sum_{i=k-h_{2}}^{k-d(k)-1}\left(h_{2}-d(k)\right) \Delta \mathbf{x}^{T}(i) \mathbf{Z}_{2} \Delta \mathbf{x}(i) \\
& -\sum_{i=k-d(k)}^{k-\tau m-1}\left(h_{2}-d(k)\right) \Delta \mathbf{x}^{T}(i) \mathbf{Z}_{2} \Delta \mathbf{x}(i) \\
& =-(1-\beta) \sum_{i=k-d(k)}^{k-\tau m-1}\left(h_{2}-\tau m\right) \Delta \mathbf{x}^{T}(i) \mathbf{Z}_{2} \Delta \mathbf{x}(i) \\
& \leq-(1-\beta) \sum_{i=k-d(k)}^{k-\tau m-1}(d(k)-\tau m) \Delta \mathbf{x}^{T}(i) \mathbf{Z}_{2} \Delta \mathbf{x}(i)
\end{aligned}
$$

Applying Lemma 1 in [22] and using (23)-(24), we have [6, 7]

$$
\begin{gathered}
-\sum_{i=k-h_{2}}^{k-\tau m-1}\left(h_{2}-\tau m\right) \Delta \mathbf{x}^{T}(i) \mathbf{Z}_{2} \Delta \mathbf{x}(i) \\
\leq-\left(\mathbf{x}(k-d(k))-\mathbf{x}\left(k-h_{2}\right)\right)^{T} \\
\quad \times \mathbf{Z}_{2}\left(\mathbf{x}(k-d(k))-\mathbf{x}\left(k-h_{2}\right)\right)
\end{gathered}
$$




$$
\begin{aligned}
& -(\mathbf{x}(k-\tau m)-\mathbf{x}(k-d(k)))^{T} \\
& \times \mathbf{Z}_{2}(\mathbf{x}(k-\tau m)-\mathbf{x}(k-d(k))) \\
& -\beta\left(\mathbf{x}(k-d(k))-\mathbf{x}\left(k-h_{2}\right)\right)^{T} \\
& \times \mathbf{Z}_{2}\left(\mathbf{x}(k-d(k))-\mathbf{x}\left(k-h_{2}\right)\right) \\
& -(1-\beta)(\mathbf{x}(k-\tau m)-\mathbf{x}(k-d(k)))^{T} \\
& \times \mathbf{Z}_{2}(\mathbf{x}(k-\tau m)-\mathbf{x}(k-d(k)))
\end{aligned}
$$

Employing (18)-(22) and (25), we have the following inequality:

$$
\Delta V(k) \leq \zeta^{T}(k)\left[(1-\beta) \Psi_{1}+\beta \Psi_{2}\right] \zeta(k)
$$

Since $0 \leq \beta \leq 1, \Delta V(k)<0$ for all nonzero $\zeta(k)$ if (5) hold true. This completes the proof of Theorem 1 .

Remark 2. To prove Theorem 1, a methodology similar to [6, 7] has been adopted.

Remark 3. It may be noted that the inequalities (5) are LMIs and can be effectively solved by using MATLAB LMI toolbox $[31,32]$.

Remark 4. For a given $\tau$ and $m$, the allowable maximum value of $h_{2}$ for guaranteeing the global asymptotic stability of system (1)-(4) can be obtained by iteratively solving (5).

Remark 5. For $m=1$, after some algebraic manipulations, it can be shown that Theorem 1 is equivalent to Theorem 1 in [7]. The parameters $m$ and $\tau$ (subject to (4)) in Theorem 1 represent some additional degrees of freedom, that is, in comparison with Theorem 1 in [7] which is free of these parameters.

Remark 6. A comparison of the number of the decision variables involved in several recent stability results is summarized in Table 1. It may be observed that the size of complexity in $[6,7,18,19,23]$ is only related to state dimension $n$, whereas the complexity of [29, Theorem 3.1], [30, Theorem 2], and Theorem 1 depends on both $n$ and $m$.

The total number of scalar decision variables of Theorem 1 is $D_{1}=(n / 2)\left[n\left(m^{2}+5\right)+(m+5)\right]$, and the total row size of the LMIs is $L_{1}=n(3 m+11)$. The numerical complexity of Theorem 1 is proportional to $L_{1} D_{1}^{3}$ [14]. The total number of scalar decision variables of Theorem 2 in [30] is $D_{2}=$ $(n / 2)\left[n\left(3 m^{2}+18 m+41\right)+(3 m+11)\right]$, the total row size of the LMIs is $L_{2}=n(7 m+31)$, and the numerical complexity is proportional to $L_{2} D_{2}^{3}$. Therefore, Theorem 1 has much smaller numerical complexity than Theorem 2 in [30]. Since no free weighting matrix has been introduced in the presented method, Theorem 1 involves less number of decision variables as compared to Theorem 3.1 in [29] (see Table 1). Thus, in general, Theorem 1 is numerically less complex than Theorem 3.1 in [29].
TABLE 1: Comparison of the number of decision variables involved in various methods.

\begin{tabular}{lc}
\hline Methods & Number of the decision variables \\
\hline Theorem 1 in [19] & $\left(23 n^{2}+5 n\right) / 2$ \\
Theorem 3 in [19] & $\left(67 n^{2}+9 n\right) / 2$ \\
Theorem 1 in [23] & $9 n^{2}+3 n$ \\
Theorem 1 in [18] & $13 n^{2}+5 n$ \\
Proposition 2 in [6] & $3 n^{2}+3 n$ \\
Theorem 1 in [7] & $3 n^{2}+3 n$ \\
Theorem 2 in [30] & $(n / 2)\left[n\left(3 m^{2}+18 m+41\right)+(3 m+11)\right]$ \\
Theorem 3.1 in [29] & $(n / 2)\left[n\left(m^{2}+4 m+19\right)+(m+5)\right]$ \\
Theorem 1 & $(n / 2)\left[n\left(m^{2}+5\right)+(m+5)\right]$ \\
\hline
\end{tabular}

Remark 7. Using similar steps as in the proof of Proposition 8 in [30], it is easy to establish that the conservatism of the stability result obtained via Theorem 1 is nonincreasing as the number of partitions increases.

\section{Extensions to Uncertain State-Delayed Discrete-Time Systems}

In this section, we extend the previously discussed approach to derive global asymptotic stable conditions for delayed discrete-time systems with norm-bounded uncertainties.

Consider the system given by

$$
\mathbf{x}(k+1)=(\mathbf{A}+\Delta \mathbf{A}) \mathbf{x}(k)+\left(\mathbf{A}_{1}+\Delta \mathbf{A}_{1}\right) \mathbf{x}(k-d(k)) .
$$

The parameter uncertainties $\Delta \mathbf{A}$ and $\Delta \mathbf{A}_{1}$ are assumed to be norm-bounded and of the following form:

$$
\left[\begin{array}{ll}
\Delta \mathbf{A} & \Delta \mathbf{A}_{1}
\end{array}\right]=\mathbf{H F}\left[\begin{array}{ll}
\mathbf{E}_{0} & \mathbf{E}_{1}
\end{array}\right],
$$

where $\mathbf{H} \in \mathbf{R}^{n \times q}$ and $\mathbf{E}_{i} \in \mathbf{R}^{p \times n}(i=0,1)$ are known constant matrices and $\mathbf{F} \in \mathbf{R}^{q \times p}$ is an unknown matrix which satisfies

$$
\mathbf{F}^{T} \mathbf{F} \leq \mathbf{I}
$$

The parameter uncertainties $\Delta \mathbf{A}$ and $\Delta \mathbf{A}_{1}$ are said be admissible if both (28) and (29) are satisfied. result.

The following lemma is needed in the proof of our next

Lemma $\mathbf{8}$ (see $[5,25]$ ). Let $\boldsymbol{\Sigma}, \boldsymbol{\Gamma}, \mathbf{F}$, and $\mathbf{M}$ be the real matrices of appropriate dimensions with $\mathbf{M}$ satisfying $\mathbf{M}=\mathbf{M}^{T}$; then

$$
\mathbf{M}+\mathbf{\Sigma} \mathbf{F} \Gamma+\Gamma^{T} \mathbf{F}^{T} \boldsymbol{\Sigma}^{T}<\mathbf{0}
$$

for all $\mathbf{F}^{T} \mathbf{F} \leq \mathbf{I}$, if and only if there exists a scalar $\varepsilon>0$ such that

$$
\mathbf{M}+\varepsilon^{-1} \boldsymbol{\Sigma} \boldsymbol{\Sigma}^{T}+\varepsilon \boldsymbol{\Gamma}^{T} \boldsymbol{\Gamma}<\mathbf{0}
$$

Theorem 9. For given scalars $h_{1}$ and $h_{2}$, system described by (27)-(29) and (2)-(4) is globally asymptotically stable for all the admissible uncertainties, if there exist real matrices 
TABLE 2: Comparison of the maximum values of $h_{2}$ for given $h_{1}$.

\begin{tabular}{lccccc}
\hline Methods & $h_{1}=4$ & $h_{1}=6$ & $h_{1}=10$ & $h_{1}=12$ & 13 \\
\hline Theorem 1 in [20] & 8 & 9 & 12 & 16 & 20 \\
Theorem 1 in [19] & 13 & 14 & 15 & 19 & 22 \\
Proposition 2 in [6] & 15 & 16 & 18 & 19 & 25 \\
Theorem 1 in [7] & 15 & 16 & 18 & 25 \\
Theorem 1 & $15(m=1, \tau=4)$ & $16(m=1, \tau=6)$ & $18(m=1, \tau=10)$ & $19(m=1, \tau=12)$ & $25(m=1, \tau=20)$ \\
& $15(m=2, \tau=2)$ & $16(m=2, \tau=3)$ & $18(m=2, \tau=5)$ & $20(m=2, \tau=6)$ & $26(m=2, \tau=10)$ \\
\hline
\end{tabular}

TABLE 3: Comparison of upper bound $\bar{\alpha}$ for given $h_{1}$ and $h_{2}$.

\begin{tabular}{lcccccc}
\hline Methods & $2 \leq d(k) \leq 7$ & $3 \leq d(k) \leq 9$ & $5 \leq d(k) \leq 10$ & $6 \leq d(k) \leq 12$ & $10 \leq d(k) \leq 15$ & $20 \leq d(k) \leq 26$ \\
\hline Theorem 5 in [20] & 0.0830 & Infeasible & Infeasible & Infeasible & Infeasible & Infeasible \\
Corollary 2 in [19] & 0.1901 & 0.1457 & 0.1313 & 0.0906 & 0.0655 & Infeasible \\
Theorem 4 in [7] & 0.1920 & 0.1548 & 0.1425 & 0.1146 & 0.1023 & Infeasible \\
& & & & 0.1194 & 0.1080 & $(m=2, \tau=10)$ \\
Theorem 9 & 0.1927 & 0.1560 & 0.1476 & 0.1194 & $(m=2, \tau=5)$ & 0.0837 \\
& $(m=2, \tau=1)$ & $(m=3, \tau=1)$ & $(m=5, \tau=1)$ & $(m=2, \tau=3)$ & $(m=5, \tau=4)$ \\
\hline
\end{tabular}

$\mathbf{P}=\mathbf{P}^{T}>\mathbf{0}, \mathbf{Q}_{i}=\mathbf{Q}_{i}^{T}>\mathbf{0}(i=1,2,3)$, and $\mathbf{Z}_{i}=\mathbf{Z}_{i}^{T}>$ $\mathbf{0}(i=1,2)$ and a scalar $\varepsilon>0$ such that the following LMIs hold:

$$
\begin{aligned}
& {\left[\begin{array}{cccc}
\overline{\mathbf{\Psi}}_{1}+\varepsilon \overline{\mathbf{E}}^{T} \overline{\mathbf{E}} & \boldsymbol{\Delta}_{1}^{T} \mathbf{P} & \boldsymbol{\Delta}_{2}^{T} \overline{\mathbf{Z}} & \mathbf{0}_{(m+3) n \times q} \\
\mathbf{P} \boldsymbol{\Delta}_{1} & -\mathbf{P} & \mathbf{0}_{n} & \mathbf{P} \mathbf{H} \\
\overline{\mathbf{Z}} \boldsymbol{\Delta}_{2} & \mathbf{0}_{n} & -\overline{\mathbf{Z}} & \overline{\mathbf{Z}} \mathbf{H} \\
\mathbf{0}_{q \times(m+3) n} & \mathbf{H}^{T} \mathbf{P} & \mathbf{H}^{T} \overline{\mathbf{Z}} & -\varepsilon \mathbf{I}_{q}
\end{array}\right]<\mathbf{0},} \\
& {\left[\begin{array}{cccc}
\overline{\mathbf{\Psi}}_{2}+\varepsilon \overline{\mathbf{E}}^{T} \overline{\mathbf{E}} & \boldsymbol{\Delta}_{1}^{T} \mathbf{P} & \boldsymbol{\Delta}_{2}^{T} \overline{\mathbf{Z}} & \mathbf{0}_{(m+3) n \times q} \\
\mathbf{P} \boldsymbol{\Delta}_{1} & -\mathbf{P} & \mathbf{0}_{n} & \mathbf{P} \mathbf{H} \\
\overline{\mathbf{Z}} \boldsymbol{\Delta}_{2} & \mathbf{0}_{n} & -\overline{\mathbf{Z}} & \overline{\mathbf{Z}} \mathbf{H} \\
\mathbf{0}_{q \times(m+3) n} & \mathbf{H}^{T} \mathbf{P} & \mathbf{H}^{T} \overline{\mathbf{Z}} & -\varepsilon \mathbf{I}_{q}
\end{array}\right]<\mathbf{0},}
\end{aligned}
$$

where

$$
\begin{gathered}
\overline{\boldsymbol{\Psi}}_{1}=\widetilde{\boldsymbol{\Psi}}-\boldsymbol{\Lambda}_{7}^{T} \mathbf{Z}_{2} \boldsymbol{\Lambda}_{7}, \quad \overline{\boldsymbol{\Psi}}_{2}=\widetilde{\boldsymbol{\Psi}}-\boldsymbol{\Lambda}_{8}^{T} \mathbf{Z}_{2} \boldsymbol{\Lambda}_{8}, \\
\widetilde{\boldsymbol{\Psi}}=\boldsymbol{\Lambda}_{1}^{T} \widetilde{\boldsymbol{\Phi}}_{1} \boldsymbol{\Lambda}_{1}+\boldsymbol{\Lambda}_{2}^{T} \widetilde{\boldsymbol{\Phi}}_{2} \boldsymbol{\Lambda}_{2}+\boldsymbol{\Lambda}_{3}^{T} \widetilde{\boldsymbol{\Phi}}_{3} \boldsymbol{\Lambda}_{3}-\boldsymbol{\Lambda}_{4}^{T} \widetilde{\boldsymbol{\Phi}}_{4} \boldsymbol{\Lambda}_{4}-\boldsymbol{\Lambda}_{5}^{T} \mathbf{Z}_{1} \boldsymbol{\Lambda}_{5} \\
+\boldsymbol{\Lambda}_{1}^{T} \mathbf{Z}_{1} \boldsymbol{\Lambda}_{5}+\boldsymbol{\Lambda}_{5}^{T} \mathbf{Z}_{1} \boldsymbol{\Lambda}_{1}-\boldsymbol{\Lambda}_{6}^{T} \mathbf{Z}_{2} \boldsymbol{\Lambda}_{6}+\boldsymbol{\Lambda}_{3}^{T} \mathbf{Z}_{2} \boldsymbol{\Lambda}_{4}+\boldsymbol{\Lambda}_{4}^{T} \mathbf{Z}_{2} \boldsymbol{\Lambda}_{3} \\
+\boldsymbol{\Lambda}_{6}^{T} \mathbf{Z}_{2} \boldsymbol{\Lambda}_{3}+\boldsymbol{\Lambda}_{3}^{T} \mathbf{Z}_{2} \boldsymbol{\Lambda}_{6}, \\
\boldsymbol{\Lambda}_{7}=\left[\begin{array}{lll}
\mathbf{0}_{n \times m n} & \mathbf{I}_{n}-\mathbf{I}_{n} & \mathbf{0}_{n}
\end{array}\right] \\
\boldsymbol{\Lambda}_{8}=\left[\begin{array}{lll}
\mathbf{0}_{n \times(m+1) n} & \mathbf{I}_{n}-\mathbf{I}_{n}
\end{array}\right], \\
\widetilde{\boldsymbol{\Phi}}_{1}=-\mathbf{P}+\mathbf{Q}_{2}+\left(h_{2}-\tau m+1\right) \mathbf{Q}_{3}-\mathbf{Z}_{1},
\end{gathered}
$$

and $\Lambda_{i}(i=1, \ldots, 6)$ in (35) are given by (7)-(9).

Proof. Applying Theorem 1, the sufficient conditions for the global asymptotic stability of system described by (27)-(29) and (2)-(4) are obtained as follows:

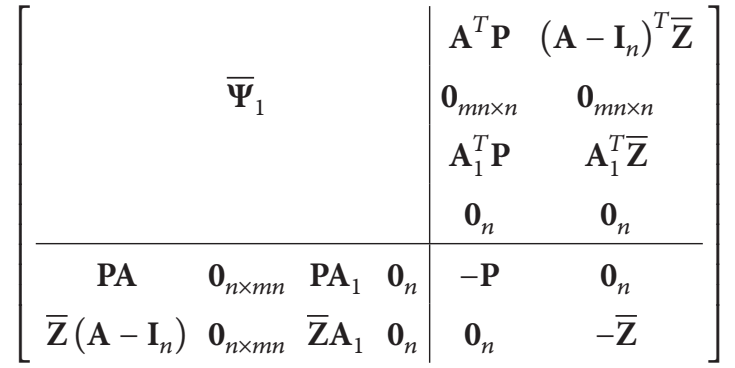

$$
\begin{aligned}
& +\left[\begin{array}{c}
\mathbf{0}_{(m+3) n \times q} \\
\mathbf{P H} \\
\overline{\mathbf{Z}} \mathbf{H}
\end{array}\right] \mathbf{F}\left[\begin{array}{llll}
\mathbf{E}_{0} & \mathbf{0}_{p \times m n} & \mathbf{E}_{1} & \mathbf{0}_{p \times 3 n}
\end{array}\right]
\end{aligned}
$$




$$
+\left[\begin{array}{c}
\mathbf{E}_{0}^{T} \\
\mathbf{0}_{m n \times p} \\
\mathbf{E}_{1}^{T} \\
\mathbf{0}_{3 n \times p}
\end{array}\right] \mathbf{F}^{T}\left[\begin{array}{lll}
\mathbf{0}_{q \times(m+3) n} & \mathbf{H}^{T} \mathbf{P} & \left.\mathbf{H}^{T} \overline{\mathbf{Z}}\right]<\mathbf{0},
\end{array}\right.
$$

$$
\begin{aligned}
& {\left[\begin{array}{cccc|cc} 
& & & & \mathbf{A}^{T} \mathbf{P} & \left(\mathbf{A}-\mathbf{I}_{n}\right)^{T} \overline{\mathbf{Z}} \\
& \bar{\Psi}_{2} & & & \mathbf{0}_{m n \times n} & \mathbf{0}_{m n \times n} \\
& & & & \mathbf{A}_{1}^{T} \mathbf{P} & \mathbf{A}_{1}^{T} \overline{\mathbf{Z}} \\
& & & & \mathbf{0}_{n} & \mathbf{0}_{n} \\
\hline \mathbf{P A} & \mathbf{0}_{n \times m n} & \mathbf{P A}_{1} & \mathbf{0}_{n} & -\mathbf{P} & \mathbf{0}_{n} \\
\overline{\mathbf{Z}}\left(\mathbf{A}-\mathbf{I}_{n}\right) & \mathbf{0}_{n \times m n} & \overline{\mathbf{Z}} \mathbf{A}_{1} & \mathbf{0}_{n} & \mathbf{0}_{n} & -\overline{\mathbf{Z}}
\end{array}\right]} \\
& +\left[\begin{array}{c}
\mathbf{0}_{(m+3) n \times q} \\
\mathbf{P H} \\
\overline{\mathbf{Z}} \mathbf{H}
\end{array}\right] \mathbf{F}\left[\begin{array}{llll}
\mathbf{E}_{0} & \mathbf{0}_{p \times m n} & \mathbf{E}_{1} & \mathbf{0}_{p \times 3 n}
\end{array}\right] \\
& +\left[\begin{array}{c}
\mathbf{E}_{0}^{T} \\
\mathbf{0}_{m n \times p} \\
\mathbf{E}_{1}^{T} \\
\mathbf{0}_{3 n \times p}
\end{array}\right] \mathbf{F}^{T}\left[\begin{array}{lll}
\mathbf{0}_{q \times(m+3) n} & \mathbf{H}^{T} \mathbf{P} & \left.\mathbf{H}^{T} \overline{\mathbf{Z}}\right]<\mathbf{0} .
\end{array}\right.
\end{aligned}
$$

By Lemma 8 and Schur complement, it can be shown that (41) and (42) are equivalent to (32) and (33), respectively. This completes the proof of Theorem 9.

Remark 10. With $m=1$, Theorem 9 reduces to an equivalent form of Theorem 4 in [7]. The parameters $m$ and $\tau$ (subject to (4)) in Theorem 9 may be treated as additional degrees of freedom, that is, in comparison with Theorem 4 in [7] which is free of such parameters.

\section{Numerical Examples}

To demonstrate the applicability of the presented results and compare them with previous results, we now consider the following examples.

Example 1 (see $[6,7,19,20,29]$ ). Consider the delayed discrete-time system (1) with the following parameters:

$$
\mathbf{A}=\left[\begin{array}{cc}
0.8 & 0 \\
0.05 & 0.9
\end{array}\right], \quad \mathbf{A}_{1}=\left[\begin{array}{cc}
-0.1 & 0 \\
-0.2 & -0.1
\end{array}\right]
$$

For given $\tau$ and $m$, the admissible maximum value of $h_{2}$ guaranteeing the asymptotically stability of the delayed discrete-time system can be obtained by solving the LMIs (5). For a given $h_{1}$, a comparison of the maximum values of $h_{2}$ calculated by different methods is summarized in Table 2 , from which one can easily see that the stability conditions obtained in this paper are less conservative than those in $[7,19,20]$.

A comparison of [29, Table 2] and Table 2 reveals that Theorem 3.1 in [29] may provide a larger upper delay bound $h_{2}$ than Theorem 1. However, as discussed in Remark 6,
Theorem 1 involves less number of decision variables as compared to Theorem 3.1 in [29].

Example 2 (see [7, 19]). Consider the following delayed discrete-time system with parameter uncertainty:

$$
\begin{aligned}
\mathbf{x}(k+1)= & {\left[\begin{array}{cc}
0.8+\alpha(k) & 0 \\
0 & 0.9
\end{array}\right] \mathbf{x}(k) } \\
& +\left[\begin{array}{cc}
-0.1 & 0 \\
-0.1 & -0.1
\end{array}\right] \mathbf{x}(k-d(k)),
\end{aligned}
$$

where $\mathbf{x}(k)=\left[\begin{array}{ll}x_{1}(k) & x_{2}(k)\end{array}\right]^{T}$ and $|\alpha(k)| \leq \bar{\alpha}$. System (44) can be represented in the form of (27) with

$$
\begin{array}{cc}
\mathbf{A}=\left[\begin{array}{cc}
0.8 & 0 \\
0 & 0.9
\end{array}\right], \quad \mathbf{A}_{1}=\left[\begin{array}{cc}
-0.1 & 0 \\
-0.1 & -0.1
\end{array}\right], \quad \mathbf{H}=\left[\begin{array}{c}
\bar{\alpha} \\
0
\end{array}\right], \\
\mathbf{E}_{0}=\left[\begin{array}{ll}
0 & 1
\end{array}\right], \quad \mathbf{E}_{1}=\left[\begin{array}{ll}
0 & 0
\end{array}\right], \quad \mathbf{F}=\frac{\alpha(k)}{\bar{\alpha}} .
\end{array}
$$

For given $h_{1}$ and $h_{2}$, we wish to find $\bar{\alpha}$ such that the uncertain system (44) is asymptotically stable for any $|\alpha(k)| \leq \bar{\alpha}$. Table 3 presents a comparison of the values of $\bar{\alpha}$ obtained by various methods. From Table 3, it is clear that Theorem 9 can provide less conservative results than $[7,19,20]$. In the situation where $20 \leq d(k) \leq 26$, the stability criteria in $[7,19,20]$ are invalid whereas the value of $\bar{\alpha}$ obtained by Theorem 9 is 0.0837 for $m=5, \tau=4$.

\section{Conclusion}

In this paper, we have considered the problem of global asymptotic stability of linear discrete-time systems with interval-like time-varying delay in the state. By utilizing the concept of delay partitioning, an LMI-based criterion for the global asymptotic stability of such systems has been established. The proposed criterion depends on both the size of delay and partition size. With the help of numerical examples, it has been illustrated that the proposed stability condition may provide less conservative result than most of the existing results $[6,7,19,20]$ due to the delay partitioning, and it may become even less conservative when the partitioning goes finer. Since no free weighting matrix has been introduced in the presented method, Theorem 1 involves less number of decision variables as compared to Theorem 3.1 in [29]. However, a comparison of [29, Table 2] and Table 2 reveals that Theorem 3.1 in [29] may provide a larger upper delay bound $h_{2}$ than Theorem 1 . The proposed criterion is numerically less complex than [30]. The method has been extended to deal with the problem of global asymptotic stability of state-delayed discrete-time systems with normbounded uncertainties.

\section{References}

[1] J. K. Hale, Theory of Functional Differential Equations, Springer, New York, NY, USA, 1977. 
[2] J. Richard, "Time-delay systems: an overview of some recent advances and open problems," Automatica, vol. 39, no. 10, pp. 1667-1694, 2003.

[3] H. Shao, "New delay-dependent stability criteria for systems with interval delay," Automatica, vol. 45, no. 3, pp. 744-749, 2009.

[4] V. K. R. Kandanvli and H. Kar, "Robust stability of discrete-time state-delayed systems employing generalized overflow nonlinearities," Nonlinear Analysis: Theory, Methods and Applications, vol. 69, no. 9, pp. 2780-2787, 2008.

[5] K. F. Chen and I. K. Fong, "Stability analysis and outputfeedback stabilisation of discrete-time systems with an interval time-varying state delay," IET Control Theory and Applications, vol. 4, no. 4, pp. 563-572, 2010.

[6] H. Shao and Q. Han, "New stability criteria for linear discretetime systems with interval-like time-varying delays," IEEE Transactions on Automatic Control, vol. 56, no. 3, pp. 619-625, 2011.

[7] H. Huang and G. Feng, "Improved approach to delaydependent stability analysis of discrete-time systems with timevarying delay," IET Control Theory and Applications, vol. 4, no. 10, pp. 2152-2159, 2010.

[8] M. S. Mahmoud, F. M. Al-Sunni, and Y. Shi, "Switched discretetime delay systems: delay-dependent analysis and synthesis," Circuits, Systems, and Signal Processing, vol. 28, no. 5, pp. 735761, 2009.

[9] M. S. Mahmoud and Y. Xia, "Robust stability and stabilization of a class of nonlinear switched discrete-time systems with timevarying delays," Journal of Optimization Theory and Applications, vol. 143, no. 2, pp. 329-355, 2009.

[10] S. Xu and J. Lam, "On equivalence and efficiency of certain stability criteria for time-delay systems," IEEE Transactions on Automatic Control, vol. 52, no. 1, pp. 95-101, 2007.

[11] E. Fridman and U. Shaked, "A descriptor system approach to $H_{\infty}$ control of linear time-delay systems," IEEE Transactions on Automatic Control, vol. 47, no. 2, pp. 253-270, 2002.

[12] Q. Han, "On stability of linear neutral systems with mixed time delays: a discretized Lyapunov functional approach," Automatica, vol. 41, no. 7, pp. 1209-1218, 2005.

[13] Q. Han, "A discrete delay decomposition approach to stability of linear retarded and neutral systems," Automatica, vol. 45, no. 2, pp. 517-524, 2009.

[14] Q. Han, "Improved stability criteria and controller design for linear neutral systems," Automatica, vol. 45, no. 8, pp. 1948-1952, 2009.

[15] Q. Han and K. Gu, "Stability of linear systems with time-varying delay: a generalized discretized lyapunov functional approach," Asian Journal of Control, vol. 3, no. 3, pp. 170-180, 2001.

[16] X. Jiang and Q. Han, "On $H_{\infty}$ control for linear systems with interval time-varying delay," Automatica, vol. 41, no. 12, pp. 2099-2106, 2005.

[17] M. Wu, Y. He, J. She, and G. Liu, "Delay-dependent criteria for robust stability of time-varying delay systems," Automatica, vol. 40, no. 8, pp. 1435-1439, 2004.

[18] Y. He, M. Wu, G. Liu, and J. She, "Output feedback stabilization for a discrete-time system with a time-varying delay," IEEE Transactions on Automatic Control, vol. 53, no. 10, pp. 23722377, 2008.

[19] H. Gao and T. Chen, "New results on stability of discrete-time systems with time-varying state delay," IEEE Transactions on Automatic Control, vol. 52, no. 2, pp. 328-334, 2007.
[20] H. Gao, J. Lam, C. Wang, and Y. Wang, "Delay-dependent output-feedback stabilisation of discrete-time systems with time varying state delay," IEE Proceedings-Control Theory and Applications, vol. 151, no. 6, pp. 691-698, 2004.

[21] E. Fridman and U. Shaked, "Stability and guaranteed cost control of uncertain discrete delay systems," International Journal of Control, vol. 78, no. 4, pp. 235-246, 2005.

[22] X. Jiang, Q. Han, and X. Yu, "Stability criteria for linear discrete-time systems with interval-like time-varying delay," in Proceedings of the American Control Conference (ACC '05), pp. 2817-2822, Portland, Ore, USA, June 2005.

[23] B. Zhang, S. Xu, and Y. Zou, "Improved stability criterion and its applications in delayed controller design for discrete-time systems," Automatica, vol. 44, no. 11, pp. 2963-2967, 2008.

[24] H. Huang and G. Feng, "State estimation of recurrent neural networks with time-varying delay: a novel delay partition approach," Neurocomputing, vol. 74, no. 5, pp. 792-796, 2011.

[25] B. Du, J. Lam, Z. Shu, and Z. Wang, "A delay-partitioning projection approach to stability analysis of continuous systems with multiple delay components," IET Control Theory and Applications, vol. 3, no. 4, pp. 383-390, 2009.

[26] Y. Zhao, H. Gao, J. Lam, and B. Du, "Stability and stabilization of delayed T-S fuzzy systems: a delay partitioning approach," IEEE Transactions on Fuzzy Systems, vol. 17, no. 4, pp. 750-762, 2009.

[27] J. Liu, B. Yao, and Z. Gu, "Delay-dependent $H_{\infty}$ filtering for Markovian jump time-delay systems: a piecewise analysis method," Circuits, Systems, and Signal Processing, vol. 30, no. 6, pp. 1253-1273, 2011.

[28] L. Wu, J. Lam, X. Yao, and J. Xiong, "Robust guaranteed cost control of discrete-time networked control systems," Optimal Control Applications and Methods, vol. 32, no. 1, pp. 95-112, 2011.

[29] P. Kokil, H. Kar, and V. K. R. Kandanvli, "Stability analysis of linear discrete-time systems with interval delay: a delaypartitioning approach," ISRN Applied Mathematics, vol. 2011, Article ID 624127, 10 pages, 2011.

[30] X. Meng, J. Lam, B. Du, and H. Gao, "A delay-partitioning approach to the stability analysis of discrete-time systems," Automatica, vol. 46, no. 3, pp. 610-614, 2010.

[31] S. Boyd, L. El Ghaoui, E. Feron, and V. Balakrishnan, Linear Matrix Inequalities in System and Control Theory, SIAM, Philadelphia, Pa, USA, 1994.

[32] P. Gahinet, A. Nemirovski, A. J. Laub, and M. Chilali, LMI Control Toolbox for Use with Matlab, MATH Works, Natick, Mass, USA, 1995. 


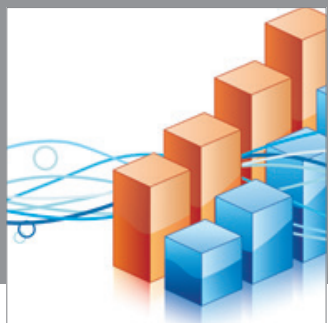

Advances in

Operations Research

mansans

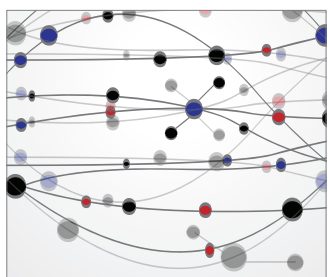

The Scientific World Journal
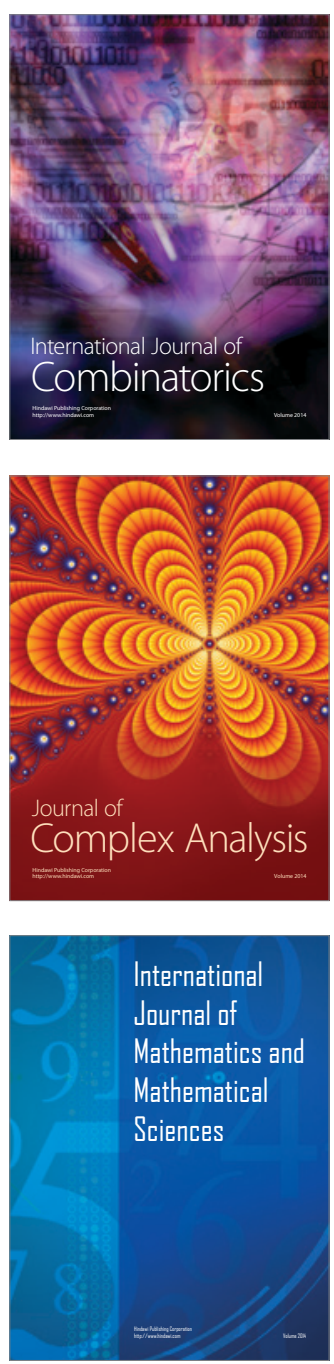
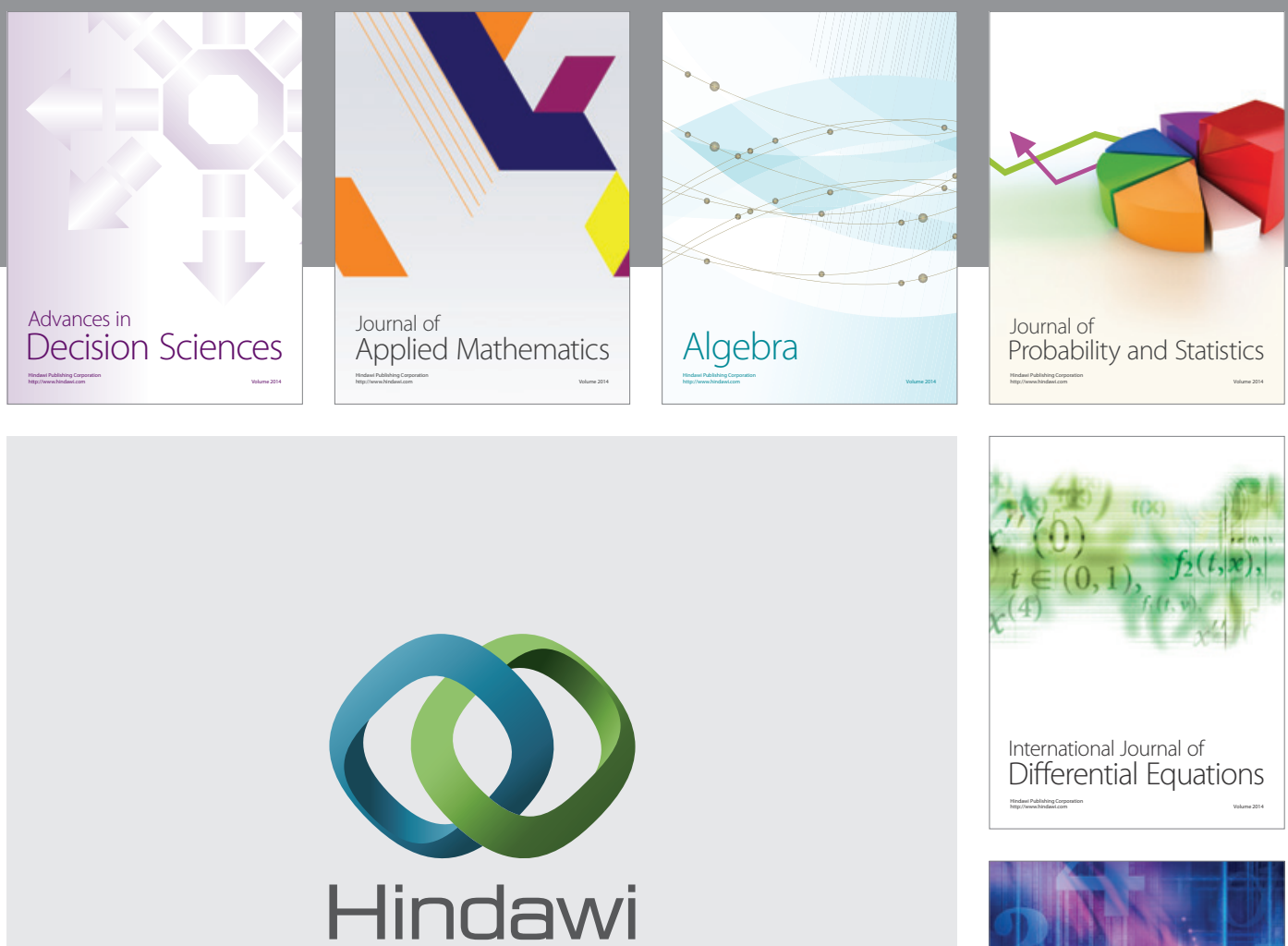

Submit your manuscripts at http://www.hindawi.com
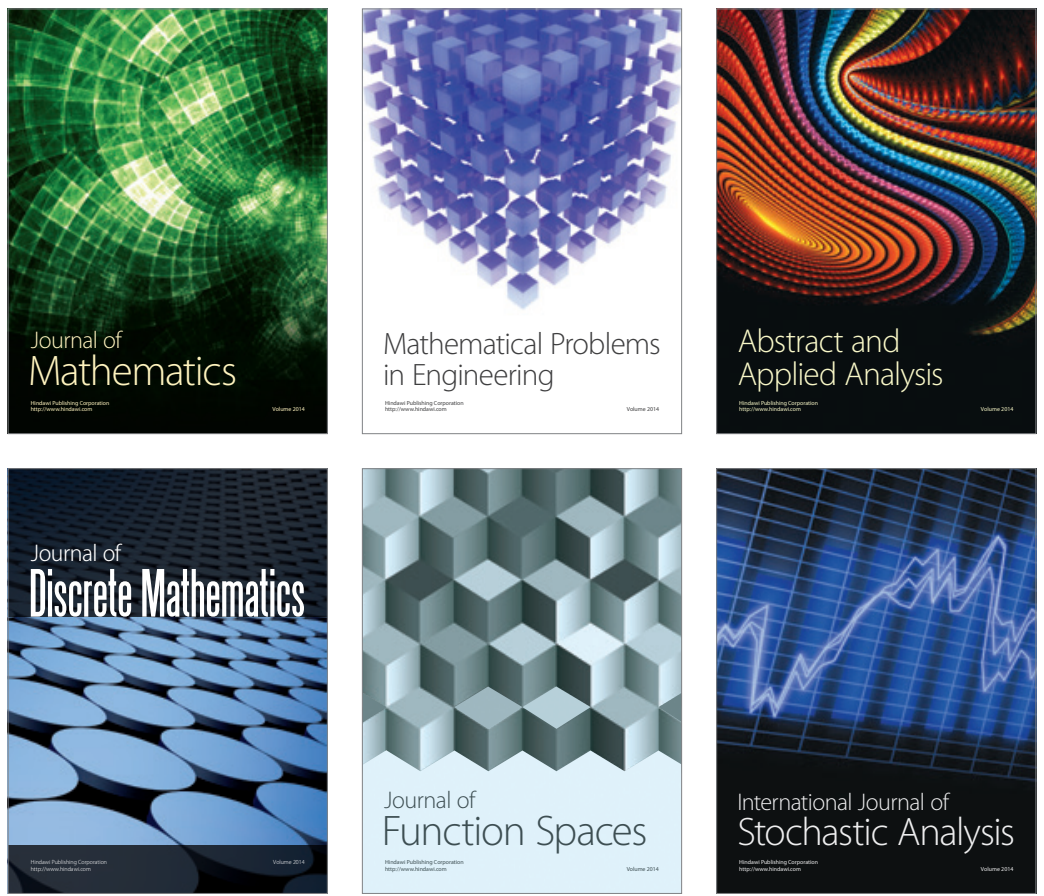

Journal of

Function Spaces

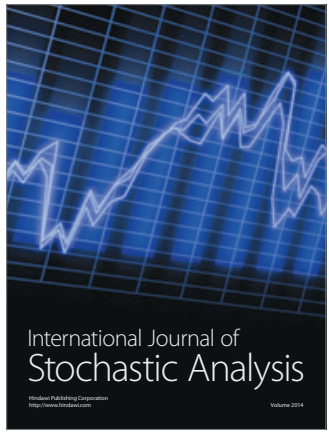

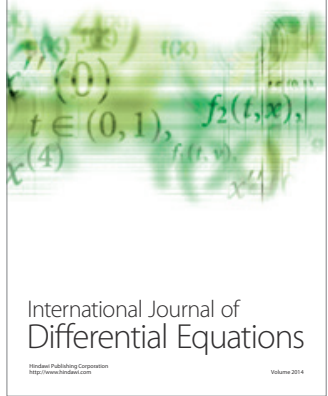
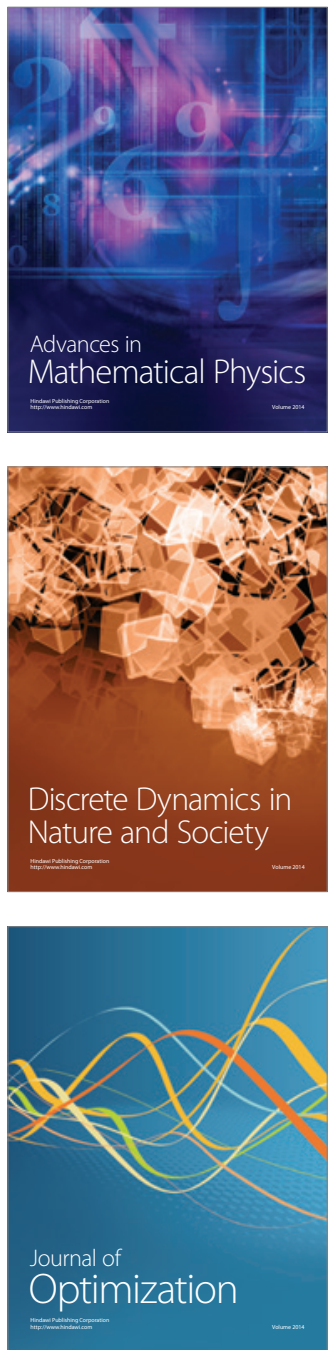